\title{
The Efficacy of Lactobacillus plantarum as Adjunctive Therapy of Bacterial Vaginosis: A Double-blind, Randomized, Placebo-controlled Trial
}

\author{
Ridha Ramadina Widiatma1, Muhammad Yulianto Listiawan', Dwi Murtiastutik ${ }^{1}$, Cita \\ Rosita Sigit Prakoeswa ${ }^{1}$, Sawitri ${ }^{1}$, Ingrid Suryanti Suryono ${ }^{2}$, Linda Astari ${ }^{1}$, Afif Nurul \\ Hidayati ${ }^{1}$ \\ ${ }^{I}$ Department of Dermatology and Venereology, Faculty of Medicine, Universitas Airlangga/Dr. \\ Soetomo General Academic Teaching Hospital, Surabaya, Indonesia \\ ${ }^{2}$ Department of Food Technology, Faculty of Engineering, Bina Nusantara University, Jakarta, \\ Indonesia
}

\begin{abstract}
Background: Bacterial vaginosis (BV) is a clinical syndrome caused by the changing of Lactobacillus spp., a producer of hydrogen peroxide, in the normal vagina with a high concentration of anaerobic bacteria, Gardnerella vaginalis and Mycoplasma hominis. This study reports a resistance of BV therapy regimens, high recurrence rates, and side effects of an antibiotic. Therefore, adjunctive therapy for BV management is needed. Research has reported the therapeutic effects of probiotic in BV; however, the results are inconsistent. Purpose: To evaluate the efficacy of standard therapy using metronidazole and Lactobacillus plantarum for BV measured by the cure rate and vaginal flora balance. Methods: A randomized, double-blind, placebo-controlled trial, 30 subjects were randomized to take metronidazole $500 \mathrm{mg}$ twice a day for seven days with weekly evaluation. The Lactobacillus plantarum or placebo group was evaluated every 4 weeks. The cure rate and vaginal flora balance were evaluated using the Amsel criteria and Nugent score before and after the intervention. Result: A total of 30 subjects returned for 28 -day follow-up, of which $60 \%$ subjects of the antibiotic/probiotic group were cured compared to $40 \%$ in the antibiotic/placebo group ( $p>0.05$ ). Conclusion: There was no difference in the increase of cure rate and vaginal flora balance between the treatment group and significant control group.
\end{abstract}

Keywords: bacterial vaginosis, probiotic, Lactobacillus plantarum, Nugent score.

Correspondence: Afif Nurul Hidayati, Department of Dermatology and Venereology, Faculty of Medicine, Universitas Airlangga/Dr. Soetomo General Academic Teaching Hospital, Jl. Mayjen Prof. Dr. Moestopo No. 6-8 Surabaya 60131, Indonesia. Phone: +62315501609, e-mail: afif_nurulhidayati@fk.unair.ac.id.

\section{BACKGROUND}

Bacterial vaginosis (BV) is a clinical syndrome as a result of the replacement of the normal hydrogen peroxide producing Lactobacillus spp. in vagina with high concentrations of anaerobic bacteria, such as Bacteroides spp., Mobiluncus spp., Gardnerella vaginalis and Mycoplasma hominis. ${ }^{1}$ Substitution of Lactobacillus spp. causes a decrease in $\mathrm{H}_{2} \mathrm{O}_{2}$ concentration. It is generally characterized by the production of gray to yellow, thin, homogeneous, fishy odor large vaginal secretions, and an increase in $\mathrm{pH}^{2}$ This clinical syndrome is also known as Haemophilus vaginalis vaginitis, Gardnerella vaginalis vaginitis, or nonspecific vaginitis. BV is the most frequent cause of complaints about vaginal body odor and vaginal discharge. However, $50 \%$ of BV patients do not aware of the symptoms. BV can provide complications in the form of urinary tract infections. The cause of microbial alteration that precipitates $\mathrm{BV}$ is not completely understood. The prevalence and distribution of BV vary among the entire world population. Some studies report a high prevalence of $\mathrm{BV}$ in African, AfroAmerican, and Afro-Caribbean racial populations. ${ }^{3}$ The BV prevalence among Indian and Indonesian women is $32 \% .{ }^{4}$ Karim et al. reported 33 new BV patients in the Sexually Transmitted Infection (STI) Division of Dr. Soetomo General Academic Teaching Hospital Surabaya from 2012 to 2014 . It was $1.35 \%$ of the patients of the STI Division and $0.1 \%$ of the total patients of the Dermatology and Venereology Outpatient Clinic of Dr. Soetomo General Academic Teaching Hospital Surabaya. ${ }^{5}$ To date, available therapeutic options serve to eliminate the signs and symptoms of vaginal infection, as well as reduce the risk of infectious complications. The standard therapy is metronidazole, clindamycin in systemic, and topical preparations. ${ }^{6}$ The drugs are commonly used in Indonesia. In the Dr. Soetomo General Academic Teaching Hospital, Surabaya, metronidazole, and clindamycin are the therapeutic choices in clinical practice guidelines for fluor albus caused by BV. ${ }^{7}$ The therapy has a low success rate, which is around $52 \%$, 
and it has a recurrence rate of around $50 \%$ in a period of 6-12 months. ${ }^{8}$ A study reported that antibiotic therapy reduces the metabolic activity of the vaginal microbiota, allowing a recurrence of BV. Several trial studies also found that strains of $G$. vaginalis and other anaerobic bacteria begin to experience resistance to metronidazole in recurrent BV cases. ${ }^{9,10}$ Some studies reported the side effects of metronidazole and clindamycin. Metronidazole has been reported to cause gastrointestinal disorders such as nausea and vomiting, metallic taste in the mouth, paresthesia, and leukopenia. The most common side effects of clindamycin are sensitivity reactions that manifest as rashes, gastrointestinal symptoms such as anorexia, abdominal pain, nausea, vomiting, diarrhea, and pseudomembranous enterocolitis. ${ }^{11}$ Considering the resistance to $\mathrm{BV}$ therapy regimens, high recurrence rates, and side effects of metronidazole and clindamycin, there is a need to look for adjunctive therapy for BV management.

Many studies have considered probiotics as additional alternative therapies for BV. ${ }^{9}$ Probiotics are living microorganisms. If they are given in sufficient quantities, they will provide beneficial health effects to the host. $^{12}$ Lactobacillus is one of the common probiotics that can be given both orally and intravenously. Long-term use of Lactobacillus is safe that it becomes an alternative for antibiotics, especially in recurrent vaginal infections. ${ }^{11}$ The mechanism of actions of Lactobacillus probiotics in BV, among others, are (1) as a barrier agent against pathogenic bacteria on the surface of the urinary epithelium, (2) maintain the low intravenous $\mathrm{pH}$ and the production of antimicrobial substances such as acids, hydrogen peroxide, and bacteriocin, (3) polyamine degradation, and (4) surfactant production which functions as an anti-adhesive agent. ${ }^{13}$ Normal vaginal microflora plays an important role as a defense and protection against urogenital infections. There are several species of Lactobacillus found in the vagina, one of which is Lactobacillus plantarum. This study used L. plantarum IS-10506, a probiotic isolated from the curd of fermented traditional Indonesian buffalo milk. $L$. plantarum IS-10506 has the potential as an appropriate probiotic for Indonesians, and it has often been exposed to contaminant and pathogenic bacteria in Indonesia. ${ }^{14}$ L. plantarum IS-10506 exhibits a high ability in adhering to hydrocarbon compounds, and it can auto-aggregate. L. plantarum IS-10506 from the curd can adhere or attach well to mucosal epithelial cells. ${ }^{15}$ Various scientific literature shows that several strains of Lactobacillus have been published as antipatogen probiotics. The Lactobacillus strains are $L$. rhamnosus GG, L. acidophilus, L. casei Shirota, L. reuteri, L. casei, L. rhamnosus, L. fermentum, $L$. johnsonii, and L. plantarum. ${ }^{14}$ Mastromarino mentioned that vaginal tablets containing a combination of three types of Lactobacillus, namely Lactobacillus brevis (CD2), Lactobacillus salivarius (FV2) and Lactobacillus plantarum (FV9) have been used and developed for prophylaxis and treatment of vaginal infections. L. salivarius and L. plantarum strains produce anti-infective agents, including hydrogen peroxide, and they can compete competitively with other vaginal pathogenic microorganisms. Overall strains can form temporary intravaginal colonies within 5 days after therapy. ${ }^{16}$ Anukam's research in 2006 concluded that an oral administration of probiotics containing Lactobacillus rhamnosus GR-1 and Lactobacillus reuteri $R C-14$ combined with metronidazole therapy regimens had a better result than metronidazole and placebo therapy in BV patients. Primary outcome was the cure of BV as determined by normal Nugent score, negative sialidase test, and no symptoms or signs of BV on day $30.88 \%$ of the subjects were cured in the antibiotic/probiotic group compared to the $40 \%$ of subjects in the antibiotic/placebo group $(p<0.001) .{ }^{17}$ The heterogeneity of the products and probiotic strains used, methodology, and research results have not provided sufficient evidence to recommend probiotics as an adjunctive BV therapy; therefore, further research in controlled clinical studies is needed to evaluate the effectiveness of these probiotic strains. To date, no studies are investigating the effectiveness of $L$. plantarum probiotics in BV in Indonesia.

We aimed to conduct an experimental, analytical study using a double-blind, randomized controlled clinical trial method comparing the L. plantarum probiotic therapy and placebo in BV patients in the STI Division of Dermatology and Venereology Outpatient Clinic Dr. Soetomo General Academic Teaching Hospital, Surabaya. The provision of standard therapy using metronidazole and probiotics is expected to restore the balance of normal vaginal flora, thereby increasing the cure rate of $\mathrm{BV}$. We also expected that this study could serve as a reference to improve the clinical practice of fluor albus caused by BV and the basis for further research. 


\section{METHODS}

This was a double-blind, randomized controlled clinical trial using a placebo. The study population was all BV patients of the STI Division of Dermatology and Venereology Outpatient Clinic Dr. Soetomo General Academic Teaching Hospital Surabaya. Subjects were BV patients aged more than 15 years old who fulfilled the diagnostic criteria for $\mathrm{BV}$ according to Amsel criteria, were in good general condition, and were willing to participate in the study. The exclusion criteria were patients taking antibiotics in the past week, patients taking probiotics in the last 4 weeks, patients having a history of allergies to antibiotics and probiotics used in the study. The sampling technique was consecutive sampling, with a sample size of 30 . The research subjects were then divided into two groups randomly to get standard therapy of metronidazole and L. plantarum (15 subjects) or standard therapy of metronidazole and placebo (15 subjects). Simple randomization was performed to determine the L. plantarum group and the control group.

The study begun by admissioning BV patients who come for anamnesis and gram staining from vaginal swab continued with Amsel criteria. Per the Amsel criteria, a BV diagnosis is established if 3 out of 4 criteria are positive. The criteria are (1) homogeneous, thin, grayish-white vaginal discharge attached to the vaginal wall, (2) clue cells in wet preparations ( $>20 \%$ of total vaginal epithelium seen on microscopic examination with physiological $\mathrm{NaCl}$ and 100 times enlargement), (3) a positive amine test that is, a fishy odor on the vaginal discharge with $10 \%$ potassium hydroxide drops (Whiff test), and (4) vaginal $\mathrm{pH}$ of more than 4.5 . The subjects with clinical symptoms were then examined to determine the Nugent score per gram staining of vaginal discharge results. A shift in bacterial morphotype from dominant Lactobacillus to Gardnerella dominant and anaerobic bacteria was noted. The vaginal flora abnormality scale is divided into normal (0-3), intermediate (4-6), and bacterial vaginosis $(7-10)$.

The research subjects were then randomly grouped into L. plantarum group and placebo group after their basic data are recorded. The L. plantarum group received L. plantarum 2 x 1 capsule per day orally, and the placebo group received placebo $2 \times 1$ capsule per day in the placebo. Each group received the treatment for 4 weeks. The clinical manifestation by Amsel criteria and Nugent was then scored at the end of week 1, 2, and 4 . The treatment group received the standard therapy using metronidazole 2 x $500 \mathrm{mg}$ for 7 days and evaluated weekly during the follow-up visit. If the Amsel criteria and Nugent score suggest that the subject has been cured, the metronidazole therapy is then stopped.

The drug packaging and randomization were carried out by the Pharmacy Department of Dr. Soetomo General Academic Teaching Hospital Surabaya. The randomization records were stored in the pharmacy and were not opened until the completion of the study. The researchers and research subjects did not know what drugs were given to the research subjects.

Each package contained L. plantarum powder with a dose of $950 \mathrm{mg}\left(0.9 \times 10^{9} \mathrm{CFU}\right)$. The placebo package consisted of a mixture of skim milk and avicel (cellulose) made in such a way to mimic L. plantarum in terms of shape, packaging, odor, color, and taste. The L. plantarum and placebo packages were provided by the Faculty of Engineering, Department of Food Technology, Bina Nusantara University, Jakarta. This study has been reviewed by the ethics committee of Dr. Soetomo General Academic Teaching Hospital.

\section{RESULT}

The age of research subjects varied, and the largest age group was 26-35 years (46.2\%). The youngest participant was 20 years old, and the oldest was 54 years old. The education level of most subjects was high school graduate $(53.3 \%)$. Most of the subjects were housewives (56.7\%). 27 out of 30 subjects (90\%) were married. Table 1 presents the subjects' sociodemographic characteristics.

The BV clinical manifestation of the 30 research subjects was quite homogeneous. Some subjects showed more than one complaint and clinical manifestation. The main complaint was grayish white discharge, as reported in 23 subjects (76.7\%) followed by abnormal vaginal odor (fishy odor), as reported in 7 subjects $(23.3 \%)$. This study was obtained several periods and frequency of complaints of research subjects. The most common complaint period was 114 days $(66.7 \%)$, and the least common complaint period was more than 30 days (6.7\%). 27 subjects (90\%) experienced BV for the first time, and 3 subjects (10\%) had recurrent BV. Table 2 presents the distribution of chief complaints, the period of $\mathrm{BV}$, and frequency of complaint.

The most common predisposing factor was active sexual relations found in 26 subjects $(86.7 \%)$. The subjects claimed that they 3-4 sexual intercourses per 
week. The precipitating factors were vaginal douching (46.7\%) and hormonal and intrauterine device contraceptives (36.7\%). A participant (3.3\%) was also reported having multiple sexual partners. In this study, there was no pregnant research. We found that 6 subjects $(20 \%)$ had a history of STI, and 3 subjects $(10 \%)$ had a history of recurrent BV. There has no universally accepted definition of recurrent BV. However, few publications proposed 3 or more BV (clinically by Amsel criteria or microscopically) per 12 months as recurrent $\mathrm{BV}$.

In the probiotic group, 9 subjects $(60 \%)$ recovered and had vaginal flora balance within the normal category, 5 subjects $(33.3 \%)$ recovered and had vaginal flora balance within the intermediate category, and 1 participant $(6.7 \%)$ did not recover and had vaginal flora balance within an abnormal category or bacterial vaginosis. In the placebo group, 6 subjects $(40 \%)$ recovered and had vaginal flora balance within the normal category, 8 subjects $(53.3 \%)$ recovered and had vaginal flora balance within the intermediate category, and 1 participant (6.7\%) did not recover and had vaginal flora balance within an abnormal category or bacterial vaginosis.

Table 1 Sociodemographic characteristics of the research subjects

\begin{tabular}{llcc}
\hline \multicolumn{1}{c}{ Category } & & Classification & Total \\
\hline Age (years) & $15-25$ & 5 & 16.7 \\
& $26-35$ & 10 & 33.3 \\
& $36-45$ & 7 & 23.3 \\
& $46-55$ & 8 & 26.7 \\
& $>55$ & 0 & 0 \\
\hline Education level & Elementary school & 2 & 6.7 \\
& Junior high school & 3 & 10.0 \\
& Senior high school & 16 & 53.3 \\
& Bachelor's degree & 9 & 30.0 \\
\hline Occupation & Unemployed & 1 & 3.3 \\
& Government employee & 1 & 3.3 \\
& Private employee & 11 & 36.7 \\
& Housewife & 17 & 56.7 \\
\hline Marital status & Married & 27 & 90.0 \\
& Unmarried & 3 & 10.0 \\
\hline
\end{tabular}

Table 2. Distribution of chief complaint, the period of BV and frequency

\begin{tabular}{lcc}
\hline Category & Amount & $\%$ \\
\hline Chief complaint* & & 76.7 \\
White grayish vaginal discharge & 23 & 23.3 \\
Abnormal vaginal odor (fishy odor) & 7 & 66.7 \\
Period of BV (days) & 20 & 26.7 \\
$1-14$ & 8 & 6.6 \\
$15-30$ & 2 & 90.0 \\
$>30$ & & 10.0 \\
Frequency & 27 & 3 \\
First time & 3 & \\
Recurrent & & \\
\hline
\end{tabular}

*Subject can have more than one component

$\mathrm{BV}=$ Bacterial vaginosis. 
Table 3. The distribution of predisposing factor and history of illness

\begin{tabular}{lcc}
\hline Category & Amount & $\%$ \\
\hline Predisposing factor & 0 & 0 \\
$\quad$ Pregnancy & 14 & 46.7 \\
Douching & 26 & 86.7 \\
Active sexual behavior & 1 & 3.3 \\
Have multiple sexual partners & 11 & 36.7 \\
Use of contraception (hormonal and intrauterine device) & 3 & 10.0 \\
History of sexual partner with discharge & & 20.0 \\
History of illness & 6 & 10.0 \\
$\quad$ History of STI & 3 & \\
History of BV before & &
\end{tabular}

$\mathrm{STI}=$ Sexually transmitted infection; $\mathrm{BV}=$ Bacterial vaginosis.

Tabel 4. The cure rate and vaginal flora balance

\begin{tabular}{lcccc}
\hline \multicolumn{5}{c}{ Cure Status } \\
\hline Group & Cured (Normal) & Intermediate & Not cured (BV) & $\mathrm{p}$ \\
\hline Probiotic & 9 & 5 & 1 & 0.925 \\
Placebo & 6 & 8 & 1 & \\
\hline Total & 15 & 13 & 2 & \\
\hline
\end{tabular}

$\mathrm{BV}=$ Bacterial vaginosis.

The comparison of cure rate and vaginal flora balance between the probiotic and placebo group before and after treatment was calculated using the Kolmogorov-Smirnov test. The test result of the cure rate and vaginal flora balance between the groups showed no difference $(\mathrm{p}=0.925)$.

\section{DISCUSSIONS}

The cure rate and vaginal flora balance were assessed using the Nugent score and the Amsel criteria. Subjects with the Nugent a score of 0-3 were declared cured and had vaginal flora balance within the normal category, those with a score of 4-6 were declared cured had vaginal flora balance within the intermediate category, and those with a score of 7-10 were declared not cured and had vaginal flora balance within the abnormal category or bacterial vaginosis. Each participant was assessed using Nugent scores at week $0,1,2$, and 4 . The probiotic group had a higher recovery percentage than the placebo group, $60 \%$ and $40 \%$. This showed that the standard metronidazole therapy combined with L. plantarum probiotics could improve the clinical cure rate of $\mathrm{BV}$ treatment. However, the Kolmogorov-Smirnov test result on the cure status and vaginal flora balance showed no significant difference.

There was one unrecovered participant $(6.7 \%)$ in each group. Some studies have assessed various antibiotic protocols for BV therapy in Caucasian, African, American, European, Asian and Mexican women with a treatment failure rate of $39 \% .{ }^{17}$ Factors that cause the failure of BV therapy, among others, are antibiotic resistance to the bacteria causing $\mathrm{BV}$ and medication compliance. Metronidazole is still the firstline drug in BV therapy, and it is said to have a fairly low level of resistance to pathogenic bacteria in BV. However, some recent studies reported that $G$. vaginalis strains, which is the main bacteria causing $\mathrm{BV}$, experiences resistance to metronidazole. ${ }^{18}$ In this study, we did not examine the subjects' bacterial culture and antibiotic sensitivity. Thus, no antibiotic resistance data collected. Medication compliance also plays an important role in influencing the cure rate. Failure to comply with the prescribed medication was suspected to be the cause of unrecovered subjects.

A systematic review of 5 studies on various probiotic strains in $\mathrm{BV}$ reported that a combination of probiotics and antibiotics reduced the risk of $\mathrm{BV}$ recurrence by half compared to antibiotic therapy alone ( $R R=0.49 ; 95 \%$ CI: 0.17-1.44). Each probiotic strain is a specific strain that the outcome depends on the type and dose of probiotics given. ${ }^{9}$ In terms of probiotics, various factors can affect the results of studies. The mechanism of action of probiotic bacteria is highly dependent on the strain of the bacterium. Furthermore, it should be noted that two bacteria with the same species could result differently as the genetic of each bacteria varies. Variation in number of doses, duration of probiotics intake, type of strain used, single strain or multistrain probiotics, a combination of probiotics and prebiotics, and different probiotic formula can also affect the results of the study. 
A meta-analysis study evaluated 10 RCTs regarding the effectiveness and safety of probiotics in BV therapy. The study compared the probiotic therapy with placebo control and the antibiotic therapy and reported that most subjects in the probiotic group experienced clinical improvement on day $30(\mathrm{RR}=$ 2.57; 95\% CI: 1.96-3.37). The effect began to decrease but remained significant after 8 weeks, and no side effect reported. Wang concluded that probiotics are safe to use and provide short and long term benefits in the management of BV. The outcomes were strainspecific. $^{19}$

Individual sensitivity to probiotic therapy might influence the result. Such sensitivity is influenced by the genetic predisposition to the probiotic response and gene susceptibility that encodes certain cytokine receptors, microbiota variations, and further affects the colonization of probiotic bacteria. Therefore, more studies are needed because the results vary greatly. ${ }^{19}$ No side effects observed. All subjects completed the treatment for 4 weeks without dropout. Several RCT studies have reported the side effects of probiotics, including flatulent, mild abdominal discomfort, nausea, and diarrhea - and all of which could improve naturally.

This study concluded that there was no significant difference in the cure rate and vaginal flora balance between the treatment groups. It was probably because the evaluation period for the probiotics was too short. Also, non-compliance in taking medication was possible. Further research is needed to evaluate the effectiveness of probiotics in recurrent $\mathrm{BV}$ and consideration to use a combination of probiotics.

\section{REFERENCES}

1. Murtiastutik D. Vaginosis bakterial. In: Barakbah J, Lumintang H, Martodihardjo S, editors. Buku ajar infeksi menular seksual. Surabaya: Airlangga University Press. 2008. P. 72-83.

2. Sharon H, Jeanne M, Holmes, KK. Bacterial vaginosis. In Holmes KK, Sparling PF, Stamm WE, Piot P, Wasserheit JN, Corey L, et al., editors. Sexually transmitted disease 4 th ed. New York: McGraw Hill. 2008. P. 737-68.

3. Kenyon C, Colebunders R, Crucitti T. The global epidemiology of bacterial vaginosis: a systematic review. Am J Obstet Gynecol 2013; 209(6): 50523.

4. Kementrian Kesehatan Republik Indonesia. Vaginosis bakterial. In: Pedoman praktis diagnosis \& tatalaksana infeksi menular seksual. Jakarta: Kementrian Kesehatan RI Direktorat Jendral Pengendalian Penyakit dan Penyehatan Lingkungan; 2015. P. 20-1.
5. Karim A, Barakbah J. Bacterial vaginosis. BIKKK 2017;28(3): 235-42.

6. Centers for disease control and prevention (CDC). Sexually Transmitted Diseases Treatment Guidelines, MMWR. 2015: 69-72.

7. RSUD Dr. Soetomo. Panduan praktik klinis (PPK) Fluor albus. 2014. p. 107-9.

8. Stemmet M, Africa CW, editors. Proceedings of The World Conference on Biological and Biomedical Science; 2013 Sep 23-27; California, USA. Los Angeles:Peertechz; 2015.

9. Darmayanti AT, Murti B, Susilawati TN. The effectiveness of adding probiotic on antimicrobial for bacterial vaginosis: a systematic review. Indonesian Journal of Medicine 2017; 2(3): 1618.

10. Ya W, Reifer C, Miller LE. Efficacy of vaginal probiotic capsules for recurrent bacterial vaginosis: a double-blind, randomized, placebocontrolled study. Am J Obstet Gynecol 2010; 203 : 120.e1-6.

11. Vujic, G, Knez AJ, Stefanovic VD, Vrbanovic VK. Efficacy of orally applied probiotic capsules for bacterial vaginosis and other vaginal infections: a double-blind, randomized, placebocontrolled study. Eur J Obstet Gynecol 2013; 168 : 75-9.

12. Doron S, Snydman DR. Risk and safety of probiotics. Clin Infect Dis 2015; 15(60) Suppl 2: S129-34.

13. Bastani PA, Homayouni S, Ziyadi, and VG Tabrizian. Dairy probiotic foods and bacterial vaginosis: A review on mechanism of action. In: Rigobelo EC (ed.). Probiotics. USA: Intech; 2012. p. 445-56.

14. Adib A, Wahid MH, Sudarmono P, Surono IS. Lactobacillus plantarum in stool of apparently healthy adults consuming Lactobacillus plantarum IS-10506 from Dadih. JTIP 2013; 24(2): 154-60.

15. Collado MC, Surono SI, Meriluoto J, Salminen S. Potential probiotic characteristics of Lactobacillus and Enterococcus strains isolated from traditional dadih fermented milk against pathogen intestinal colonization. J Food Prot 2017; 70(3): 700-5.

16. Mastromarino P, Vitali B, Mosca L. Bacterial vaginosis: a review on clinical trials with probiotics. The new microbiologica 2013; 36: 229-38.

17. Anukam K, Osazuwa E, Ahonkhai I, Ngwu M, Osemene G, Bruce AW, et al. Augmentation of antimicrobial metronidazole therapy of bacterial vaginosis with oral probiotic 
lactobacillus rhamnosus GR-1 and lactobacillus reuteri RC-14: randomized, double-blind, placebo-controlled trial. Microbes Infect 2006; $8: 1450-4$.

18. Schuyler JA, Mordechai E, Adelson ME, Sobel JD, Gygax SE, Hilbert DW. Identification of intrinsically metronidazole-resistant clades of gardnerella vaginalis. Diagnostic Microbiology and Infectious Disease 2016; 84(1): 1-3.

19. Wang Z, He Y, Zheng Y. Probiotic for the treatment of bacterial vaginosis: a meta-analysis. Int J Environ Res Public Health 2019; 16(20): 3859 . 